\title{
Differentiation of Market Samples of Chennai Nagakesara and Nagakesara by Chromatographic Techniques
}

\author{
SUBRAMANIAN SUBASHINI, R. KARUPPASAMY, S. THATIPELLI, RAJKUMAR SHYAMALA AND RAMACHANDRAN \\ SHAKILA* \\ Department of Chemistry, Siddha Central Research Institute (Central Council for Research in Siddha, Ministry of AYUSH, \\ Government of India), Anna Hospital Campus, Arumbakkam, Chennai 600106, Tamil Nadu, India
}

\section{Subashini et al.: Differentiation of Chennai Nagakesara and Nagakesara}

\begin{abstract}
Efficacy of any herbal drugs depends on their chemical profile and their biological activity of ingredients. The anticipated efficacy of any drug could be achieved if authenticated ingredients that are collected as per good collection practices in the appropriate season. The general problem faced in herbal drug industry is correct identification of the source plant. The present study was aimed to develop chromatographic finger print profiles by using high performance thin layer chromatography and high pressure liquid chromatography of Nagakesara and Chennai Nagakesara/Cirunakappu to record the difference between them. Both samples were procured from Chennai market, extracted successively with $n$-hexane, chloroform, ethyl acetate and ethanol extracts using Soxhlet apparatus and subjected to high performance thin layer chromatography and high pressure liquid chromatography analysis. The thin-layer chromatography of hexane extract of Nagakesara showed more spots than Chennai Nagakesara/Cirunakappu and similarly with chloroform and ethanol extracts also. Maximum number of peaks was observed in all the extracts after derivatization with vanillin sulphuric acid reagent. In the high pressure liquid chromatography results of hexane extract maximum of 22 peaks were separated in Nagakesara whereas in Chennai Nagakesara/ Cirunakappu only 14 peaks were separated; in contrast, in the ethanol extract of Chennai Nagakesara/ Cirunakappu 17 peaks were separated while in Nagakesara it was five peaks only.
\end{abstract}

Key words: Madras Nagakesara, Cirunakappu, Mesua ferrea, Mesua nagassarium, Cinammomum wightii

Siddha has encourages research efforts on scientific information about plants, plant extracts and various anatomical parts of plants as medicinal agents. Although herbal medicine exists since the dawn of time, our knowledge of how plants actually influence human physiology remains largely unexplored. This research is going on with a view to provide the phytochemical differentiation between the two plants with different analytical techniques like High-performance thin-layer chromatography (HPTLC), High-performance liquid chromatography (HPLC), Gas chromatography (GC), Gas chromatography-mass spectrometry (GC-MS), Liquid chromatography-tandem mass spectrometry (LC-MS/MS), Fourier-transform infrared spectroscopy (FTIR), Near-infrared (NIR), Raman spectra, etc.

In this communication, authors aim to differentiate the two drugs available in the crude drug market, in the name of Nagakesara. In Ayurveda, Nagakesara is equated to

*Address for correspondence

E-mail: r.shakila@gov.in

July-August 2021
Mesua ferrea (M. ferrea) Linn., whereas in classical Siddha literature, Madras Nagakesara/Cirunakappu is botanically equated as Mesua nagassarium (M. nagassarium) (Burm. f.) Kosterm ${ }^{[1]}$. But in the crude drug market, immature fruits of Cinnamomum wightii (C. wightii) Meissn are sold as Madras Nagakesara/Cirunakappu in different places of Southern $\mathrm{India}^{[2]}$. Cinnamomum ovalifolium (C. ovalifolium) Gardner ex Meisn is the synonym of C. wightii ${ }^{[3]}$. Cirunakappu is a medicinal flower bud. It is carminative, astringent, acrid, purgative, diaphoretic and aromatic. C. wightii was used for treating wounds, This is an open access article distributed under the terms of the Creative
Commons Attribution-NonCommercial-ShareAlike 3.0 License, which
allows others to remix, tweak, and build upon the work non-commercially,
as long as the author is credited and the new creations are licensed under
the identical terms

Accepted 05 August 2021

Revised 03 July 2021

Received 03 March 2020

Indian J Pharm Sci 2021;83(4):775-784 
fever, intestinal worms, headaches and menstrual problems and treat many diseases such as asthma, burning sensation in palm and soles, digestive impairment, gastric troubles, intercostal neuralgia, numbness of tongue, pleurodynia, spleen enlargement, leucorrhoea, hiccup, anemia, tuberculosis and kappa diseases. Essential oils from Cinnamomum species were isolated and screened for antimicrobial and antiinflammatory activities in our laboratory. Future studies will focus on other types of bioassays, as this process is usually considered as the first step in the discovery of new drugs $s^{[4,5]}$.

Cirunakappu finds use in many Siddha formulation viz., Amukkarac curanam ${ }^{[6-10]}$ Civataic curanam, Elatic curanam, Ilaku Cantanatit Tailam, Incic curanam, Kantaka Racayanam, Karicalai Ilakam, Narattai Ilakam, Parankippattai Iracayanam, Talicaticcuranam, Talicati vatakam, Vivati Ilakam ${ }^{[11]}$, Ilankathi Curanam, Kadukkai Ilakam, Chandrakanthi Curanam, Naavalpattai Nei, Pooranathi Ilakam, Mahavilvathi Ilakam, Milaguth Tailam, Megathennai ${ }^{[12]}$ etc. It is known as Ceylon Iron wood in English, Nagakesara in Sanskrit, Telugu and Kanada, Nagkesar in Hindi, Nakappuvu in Malayalam. Calophyllum nagassarium Burm.f. and M. nagassarium (Burm. f.) Kosterm. are synonyms to $M$. ferrea ${ }^{[13,14]}$. It is native to tropical Sri Lanka and a state tree of Tripura but it is disappearing from India. M. ferrea L. is locally known as Cobra's saffron (English), Nagakeshara (Hindi), Nagasampige (Kannada), Nageshwar (Assam), Sirunagam (Tamil). is found throughout Southeast Asia in tropical evergreen forests up to $1500 \mathrm{~m}^{\text {elevation }}{ }^{[15]}$.

Nagakesara (M. ferrea) is a medicinal stamen used in many Ayurvedic formulations viz., Bilvathi leha, Cyavanaprasa, Pugakha, Vyaghri haritaki, Eladi curna, Kalyaaka ghrta, Triphala ghrta ${ }^{[16]}$; Amrtarista, Asvagandhadyarista, Babbularista, Dasamu larista, Draks arista, Jirakady arista, Kanak asava, Kadir arista, Pippalayady arista, Punarnavady arista, Madhusnuhi rasayana, Bhaskaralavana curna, Jatiphaladi curna ${ }^{[17]}$; Haridra khanda, Narikela khanda, Arimedadi taila, Bala taila, Bhrngaraja taila, Kanaka taila ${ }^{[18]}$. The main aim of this study is to develop a chromatographic methods for the differentiation of these two plants. As these drugs are commercially sold in the Indian market under the name Nagakesara/Cirunakappu, the purpose of our investigation is to aid its importance in the field of research and as well as its commercial gradient where it might be possibly adulterated with other marketed drug like $M$. ferrae Linn. ${ }^{[19,20]}$.

\section{MATERIALS AND METHODS}

\section{Plant material:}

The plants in the name of Nagakesara/Madras, Nagakesara/Cirunakappu were purchased from Govindaraja Mudaliyar Sons Pvt. Ltd., the raw drug retail store in Chennai, Tamil Nadu and taken for the study.

\section{Solvents and chemicals:}

HPTLC pre-coated plates of silica gel $60 \mathrm{~F}_{254}$ were procured from Merck India. All the solvents were of analytical reagent (AR) grade for HPTLC and HPLC analysis procured from Merck. Vanillin from Lobacheme and sulphuric acid from Merck was laboratory reagent (LR) Grade.

\section{Preparation of extracts:}

$2 \mathrm{~g}$ of both plant samples were extracted using hexane, chloroform, ethyl acetate and ethanol successively using Soxhlet apparatus. Each extract obtained was filtered using whatman No. 1 filter paper, dried up to $5 \mathrm{ml}$ using Rotavac and these solutions were used as test solution for HPTLC analysis. Then the extracts were dried and dissolved in methanol for HPLC analysis.

\section{HPTLC instrument:}

The CAMAG $^{\circledR}$ HPTLC equipment composed of automatic Thin-layer chromatography (TLC) sampler 4 (ATS4), Visualizer and TLC scanner coupled with winCATS software, Chromatogram Immersion Device III for derivatization and TLC plate heater III.

\section{HPLC instrument:}

HPLC analysis was carried out in a modular Shimadzu LC-20 system which comprised of a LC-20AP pump, CTO-20AC column oven, a SPD-M 20A ultravioletdiode array detector (UV-DAD) detector, CBM-10A interface and LC-20 workstation was utilized. A C18 column $(250 \mathrm{~mm}$ length $\times 4.6 \mathrm{~mm}$ inner diameter $\times 5 \mu$ particle size) from Shim-pack GSIT was employed at $30^{\circ}$. Separations were done in isocratic mode.

\section{TLC/HPTLC procedure:}

\section{Sample application:}

Solvent system: The selected mobile phases for hexane extract were hexane:ethyl acetate $(6: 1, \mathrm{v} / \mathrm{v})$; 
for chloroform, ethyl acetate and ethanol extracts was toluene:ethyl acetate $(6: 4, \mathrm{v} / \mathrm{v})$.

Plate development: The samples loaded plate was kept in TLC twin trough developing chamber (after saturated with solvent vapour) with respective mobile phase and the plate was developed in the respective mobile phase up to $90 \mathrm{~mm}$.

Photo-documentation: The developed plate was dried by hot air to evaporate solvents from the plate. The plate was kept in photo-documentation chamber $\left(\mathrm{CAMAG}^{\circledR}\right.$ Visualizer) and captured the images at white light, UV $254 \mathrm{~nm}$ and UV $366 \mathrm{~nm}$.

Scanning: Before derivatization, the plate was fixed on the stage (CAMAG ${ }^{\circledR}$ SCANNER IV) and scanning was done at UV $254 \mathrm{~nm}$ and $366 \mathrm{~nm}$. The peak table, peak display and peak densitogram were noted.

Derivatization: The developed plate was sprayed with vanillin sulphuric acid as spray reagent and dried at $100^{\circ}$ in hot air oven. The plate was photodocumented in white light and scanned at UV $510 \mathrm{~nm}$ for finger print development.

\section{HPLC procedure:}

After completion of HPTLC, all the extracts were dried and then redissolved in $5 \mathrm{ml}$ of methanol, sonicated for $30 \mathrm{~min}$ and were filtered through $0.45 \mu$ membrane prior to injection. The reverse phase column $\mathrm{C} 18$ was used for chromatographic separation. The eluting solvent chosen was $100 \%$ acetonitrile with a flow rate of $1 \mathrm{ml} / \mathrm{min}$. The volume injected was $20 \mu \mathrm{l}$. The

TABLE 1: HPTLC PEAK $R_{\mathrm{f}}$ AND AREA DETAILS OF SUCCESSIVE EXTRACTS OF CN AND NK

\begin{tabular}{|c|c|c|c|c|c|c|c|c|c|c|c|c|}
\hline & \multicolumn{4}{|c|}{ UV $254 \mathrm{~nm}$} & \multicolumn{4}{|c|}{ UV 366 nm } & \multicolumn{4}{|c|}{ White light $510 \mathrm{~nm}$} \\
\hline & \multicolumn{2}{|c|}{$\mathrm{CN}$} & \multicolumn{2}{|c|}{ NK } & \multicolumn{2}{|c|}{$\mathrm{CN}$} & \multicolumn{2}{|c|}{ NK } & \multicolumn{2}{|c|}{$\mathrm{CN}$} & \multicolumn{2}{|c|}{ NK } \\
\hline & $\begin{array}{c}\operatorname{Max} \\
R_{f}\end{array}$ & Area \% & $\operatorname{Max} R_{f}$ & Area \% & $\operatorname{Max} R_{f}$ & Area \% & $\operatorname{Max} R_{f}$ & Area \% & $\operatorname{Max} R_{f}$ & Area \% & $\operatorname{Max} R_{f}$ & Area \% \\
\hline \multirow{12}{*}{$\begin{array}{l}\text { Hexane } \\
\text { extract }\end{array}$} & 0.02 & 0.81 & 0.06 & 32.15 & 0.02 & 41.43 & 0.01 & 5.07 & 0.08 & 2.39 & 0.14 & 4.39 \\
\hline & 0.13 & 6.67 & 0.14 & 11.94 & 0.07 & 10.35 & 0.05 & 11.37 & 0.12 & 40.94 & 0.22 & 11.28 \\
\hline & 0.28 & 42.34 & 0.22 & 7.78 & 0.1 & 14.82 & 0.12 & 13.71 & 0.22 & 3.4 & 0.34 & 2.9 \\
\hline & 0.38 & 9.68 & .0 .31 & 7.03 & 0.18 & 8.68 & 0.14 & 39.28 & 0.27 & 6.76 & 0.43 & 1.63 \\
\hline & 0.53 & 22.19 & 0.43 & 2.32 & 0.93 & 24.71 & 0.24 & 3.44 & 0.29 & 6.35 & 0.47 & 3.24 \\
\hline & 0.74 & 4.55 & 0.49 & 9.29 & - & - & 0.28 & 3.21 & 0.46 & 16.96 & 0.62 & 26.55 \\
\hline & 0.79 & 13.78 & 0.56 & 2.9 & - & - & 0.59 & 12.18 & 0.8 & 1.68 & 0.82 & 4.83 \\
\hline & - & - & 0.61 & 13.04 & - & - & 0.82 & 11.72 & 0.82 & 1.96 & 0.85 & 8.82 \\
\hline & - & - & 0.7 & 1.3 & - & - & - & - & 0.86 & 12.78 & 0.89 & 12.03 \\
\hline & - & - & 0.87 & 12.25 & - & - & - & - & 0.91 & 4.19 & 0.92 & 10.37 \\
\hline & - & - & - & - & - & - & - & - & 0.94 & 2.59 & 0.98 & 0.78 \\
\hline & - & - & - & - & - & - & - & - & 0.99 & 13.17 & - & - \\
\hline \multirow{16}{*}{$\begin{array}{l}\text { Chloroform } \\
\text { extract }\end{array}$} & 0.01 & 21.6 & 0.01 & 31.82 & 0.01 & 19.67 & 0.01 & 3 & 0.05 & 1.74 & 0.1 & 0.25 \\
\hline & 0.08 & 44.49 & 0.12 & 0.65 & 0.04 & 8.2 & 0.03 & 12.66 & 0.1 & 3.89 & 0.16 & 0.27 \\
\hline & 0.2 & 19.1 & 0.18 & 28.25 & 0.79 & 72.13 & 0.09 & 8.76 & 0.2 & 25.62 & 0.19 & 16.3 \\
\hline & 0.88 & 14.81 & 0.27 & 3.23 & - & - & 0.13 & 11.67 & 0.25 & 4.64 & 0.35 & 0.78 \\
\hline & - & - & 0.41 & 2.11 & - & - & 0.19 & 13.26 & 0.33 & 3.09 & 0.42 & 2.62 \\
\hline & - & - & 0.53 & 16.46 & - & - & 0.27 & 2.01 & 0.5 & 4.53 & 0.51 & 7.24 \\
\hline & - & - & 0.63 & 6.32 & - & - & 0.3 & 1.05 & 0.58 & 28.21 & 0.59 & 10.37 \\
\hline & - & - & 0.89 & 1.09 & - & - & 0.34 & 1.36 & 0.66 & 7.78 & 0.69 & 0.67 \\
\hline & - & - & 0.93 & 10.06 & - & - & 0.43 & 0.58 & 0.73 & 3.73 & 0.85 & 0.65 \\
\hline & - & - & - & - & - & - & 0.49 & 4.22 & 0.8 & 4.85 & 0.88 & 0.82 \\
\hline & - & - & - & - & - & - & 0.58 & 6.93 & 0.87 & 3.09 & 0.9 & 1.15 \\
\hline & - & - & - & - & - & - & 0.66 & 4.28 & 0.88 & 5.12 & 0.92 & 1.55 \\
\hline & - & - & - & - & - & - & 0.74 & 1.2 & 0.92 & 1.24 & 0.96 & 1.51 \\
\hline & - & - & - & - & - & - & 0.8 & 23.17 & 0.94 & 2.47 & - & - \\
\hline & - & - & - & - & - & - & 0.88 & 2.73 & - & - & - & - \\
\hline & - & - & - & - & - & - & 0.94 & 2.93 & - & - & - & \\
\hline
\end{tabular}


www.ijpsonline.com

\begin{tabular}{lcccccccccccc} 
& 0.01 & 3.85 & 0.01 & 2.93 & 0.01 & 1.9 & 0.01 & 4.8 & 0.04 & 0.27 & 0.01 & 3.41 \\
& 0.06 & 4.56 & 0.07 & 1.5 & 0.04 & 2.9 & 0.05 & 10.53 & 0.08 & 0.24 & 0.06 & 0.72 \\
& 0.13 & 1.82 & 0.1 & 0.57 & 0.09 & 5.66 & 0.11 & 5.156 & 0.11 & 0.87 & 0.08 & 2.17 \\
& 0.15 & 6.79 & 0.14 & 2.24 & 0.13 & 6.75 & 0.15 & 10.27 & 0.15 & 15.8 & 0.17 & 15.81 \\
& 0.19 & 3.38 & 0.18 & 2.14 & 0.24 & 10.41 & 0.21 & 6.4 & 0.23 & 13.49 & 0.28 & 5.12 \\
Ethyl & 0.23 & 2.6 & 0.2 & 12.62 & 0.82 & 72.37 & 0.25 & 5.24 & 0.32 & 3.95 & 0.34 & 1.55 \\
acetate & 0.25 & 10.7 & 0.29 & 6.85 & - & - & 0.32 & 1.68 & 0.41 & 2.93 & 0.37 & 4.22 \\
extract & 0.28 & 9.54 & 0.36 & 8.45 & - & - & 0.41 & 2 & 0.52 & 39.51 & 0.43 & 3.53 \\
& 0.34 & 4.43 & 0.43 & 1.05 & - & - & 0.61 & 2.87 & 0.65 & 5.63 & 0.51 & 32.85 \\
& 0.44 & 1.19 & 0.46 & 1.25 & - & - & 0.72 & 42.2 & 0.69 & 7.14 & 0.65 & 17.98 \\
& 0.55 & 31.38 & 0.53 & 26.24 & - & - & 0.85 & 8.87 & 0.79 & 0.77 & 0.76 & 7.31 \\
& 0.79 & 0.81 & 0.66 & 1.93 & - & - & - & - & 0.86 & 0.63 & 0.84 & 0.58 \\
& 0.86 & 18.96 & 0.78 & 14.37 & - & - & - & - & 0.88 & 0.94 & 0.87 & 0.39 \\
& - & - & 0.89 & 15.87 & - & - & - & - & 0.92 & 3.47 & 0.89 & 4.34 \\
& - & - & - & - & - & - & - & - & 0.95 & 4.37 & - & - \\
& 0.01 & 40.23 & 0.01 & 10.88 & 0.01 & 29.58 & 0.01 & 11.34 & 0.01 & 56.18 & 0.01 & 15.25 \\
& 0.1 & 1.44 & 0.08 & 0.33 & 0.29 & 9.8 & 0.1 & 11.74 & 0.21 & 9.75 & 0.08 & 0.46 \\
& 0.21 & 1.47 & 0.11 & 0.36 & 0.34 & 26.78 & 0.17 & 12.74 & 0.27 & 1.83 & 0.12 & 0.56 \\
& 0.26 & 8.49 & 0.14 & 1.65 & 0.52 & 18.39 & 0.28 & 9.09 & 0.4 & 0.66 & 0.17 & 0.33 \\
& 0.31 & 16.39 & 0.22 & 19.89 & 0.81 & 15.46 & 0.36 & 2.77 & 0.47 & 4.44 & 0.21 & 1.09 \\
Ethyl & 0.47 & 10.61 & 0.31 & 12.28 & - & - & 0.39 & 4.16 & 0.51 & 4.74 & 0.26 & 1.18 \\
alcohol & 0.85 & 12.54 & 0.37 & 14.37 & - & - & 0.43 & 3.93 & 0.54 & 4.87 & 0.31 & 2.7 \\
extract & 0.94 & 8.84 & 0.42 & 5.81 & - & - & 0.51 & 12.71 & 0.68 & 8.02 & 0.37 & 5.32 \\
& - & - & 0.48 & 9.45 & - & - & 0.61 & 5.11 & 0.74 & 4.8 & 0.44 & 25.8 \\
& - & - & 0.56 & 4.27 & - & - & 0.72 & 7.9 & 0.89 & 1.77 & 0.65 & 11.58 \\
& - & - & 0.65 & 1.15 & - & - & 0.78 & 12.21 & 0.94 & 1.96 & 0.73 & 6.62 \\
& - & - & 0.75 & 9.15 & - & - & 0.87 & 6.29 & 0.98 & 1 & 0.78 & 14.09 \\
& - & - & 0.85 & 10.41 & - & - & - & - & - & - & 0.92 & 15.01 \\
\hline
\end{tabular}

TABLE 2: HPLC PEAK R,AND AREA DETAILS OF DIFFERENT EXTRACTS OF CN AND NK

\begin{tabular}{|c|c|c|c|c|c|c|c|c|c|c|c|c|c|c|c|c|}
\hline \multirow{3}{*}{$\begin{array}{l}\mathrm{Pe}- \\
\mathrm{ak}\end{array}$} & \multicolumn{4}{|c|}{ Hexane extract @ 230 nm } & \multicolumn{4}{|c|}{$\begin{array}{l}\text { Chloroform extract } \\
\text { @ } 200 \mathrm{~nm}\end{array}$} & \multicolumn{4}{|c|}{$\begin{array}{l}\text { Ethyl acetate extract } \\
\text { @ } 200 \mathrm{~nm}\end{array}$} & \multicolumn{4}{|c|}{$\begin{array}{l}\text { Ethanol extract } \\
\text { @ } 230 \mathrm{~nm}\end{array}$} \\
\hline & \multicolumn{2}{|c|}{$\mathrm{CN}$} & \multicolumn{2}{|c|}{ NK } & \multicolumn{2}{|c|}{$C N$} & \multicolumn{2}{|r|}{ NK } & \multicolumn{2}{|c|}{$\mathrm{CN}$} & \multicolumn{2}{|c|}{ NK } & \multicolumn{2}{|c|}{$\mathrm{CN}$} & \multicolumn{2}{|r|}{ NK } \\
\hline & $\mathbf{R}_{\mathrm{t}}$ & $\begin{array}{c}\text { Area } \\
\%\end{array}$ & $\mathbf{R}_{\mathrm{t}}$ & $\begin{array}{c}\text { Area } \\
\%\end{array}$ & $\mathbf{R}_{\mathrm{t}}$ & $\begin{array}{c}\text { Area } \\
\%\end{array}$ & $\mathbf{R}_{\mathrm{t}}$ & $\begin{array}{c}\text { Area } \\
\%\end{array}$ & $\mathbf{R}_{\mathrm{t}}$ & $\begin{array}{c}\text { Area } \\
\%\end{array}$ & $\mathbf{R}_{\mathrm{t}}$ & $\begin{array}{c}\text { Area } \\
\%\end{array}$ & $\mathbf{R}_{\mathrm{t}}$ & $\begin{array}{c}\text { Area } \\
\%\end{array}$ & $\mathbf{R}_{\mathrm{t}}$ & $\begin{array}{c}\text { Area } \\
\%\end{array}$ \\
\hline$\overline{1}$ & 1.8 & 6.994 & 1.697 & 10.285 & 1.611 & 30.18 & 1.796 & 21.965 & 1.605 & 21.842 & 1.744 & 45.98 & 1.489 & 64.767 & 1.395 & 59.672 \\
\hline 2 & 2.052 & 13.042 & 1.954 & 8.517 & 1.859 & 6.81 & 2.044 & 28.634 & 1.699 & 42.469 & 2.316 & 16.513 & 1.758 & 14.337 & 1.562 & 59.321 \\
\hline 3 & 2.163 & 9.72 & 2.145 & 6.947 & 1.97 & 4.71 & 2.609 & 12.523 & 2.316 & 16.035 & 2.471 & 18.733 & 1.957 & 6.78 & 2.149 & 9.135 \\
\hline 4 & 2.325 & 6.783 & 2.321 & 6.664 & 2.321 & 3.23 & 3.282 & 2.25 & 4.904 & 0.929 & 4.88 & 4.955 & 2.165 & 3.16 & 2.291 & 16.766 \\
\hline 5 & 2.471 & 8.34 & 2.589 & 13.889 & 2.941 & 3.7 & 3.381 & 1.271 & 16.067 & 18.724 & +15.991 & 13.06 & 2.316 & 3.033 & 4.894 & 5.107 \\
\hline 6 & 2.634 & 4.983 & 4.894 & 15.951 & 3.526 & 18.17 & 3.55 & 3.851 & - & & 17.059 & 0.758 & 2.452 & 2.998 & - & - \\
\hline 7 & 2.741 & 6.395 & 6.27 & 3.844 & 4.497 & 2.65 & 3.811 & 5.676 & - & & - & - & 2.636 & 2.869 & - & - \\
\hline 8 & 3.352 & 6.299 & 7.415 & 1.857 & 5.788 & 10.259 & 3.936 & 23.397 & - & - & - & - & 3.9 & 1.326 & - & - \\
\hline 9 & 3.523 & 6.375 & 8.733 & 2.721 & 7.997 & 1.41 & 4.305 & 0.052 & - & - & - & - & 4.896 & 0.487 & - & - \\
\hline 10 & 3.781 & 7.975 & 10.241 & 0.619 & 10.739 & 0.3 & 4.415 & 0.223 & - & & - & - & 7.436 & 0. & - & . \\
\hline 11 & 3.9 & 16 & 11.132 & 0.671 & 11.44 & 0.236 & 5.903 & 0.157 & - & . & - & - & 7.783 & 0.016 & - & - \\
\hline 12 & 4.895 & 4.66 & 11.384 & 0.764 & 14.355 & 10.946 & - & - & - & - & - & - & 8.291 & 0.012 & - & - \\
\hline 13 & 5.27 & 1.622 & 11.915 & 1.105 & 15.416 & 7.23 & - & - & - & . & - & - & 8.759 & 0.039 & - & - \\
\hline 14 & 15.982 & 0.712 & 13.581 & 0.318 & - & . & - & - & - & - & - & - & 10.268 & 0.014 & - & - \\
\hline 15 & - & - & 14.631 & 2.034 & - & - & - & - & - & - & - & - & 16.057 & 0.052 & - & - \\
\hline 16 & - & - & 15.242 & 2.643 & - & - & - & - & - & - & - & - & 17.255 & 0.024 & - & - \\
\hline 17 & - & - & 16.02 & 14.627 & - & - & - & - & - & - & - & - & 19.966 & 0.067 & - & - \\
\hline 18 & - & - & 17.097 & 2.301 & - & - & - & - & - & - & - & - & - & - & - & - \\
\hline 19 & - & - & 19.915 & 1.942 & - & - & - & - & - & - & - & - & - & - & - & - \\
\hline 20 & - & - & 21.565 & 0.629 & - & - & - & - & - & - & - & - & - & - & - & - \\
\hline 21 & - & - & 22.504 & 0.818 & - & - & - & - & - & - & - & - & - & - & - & - \\
\hline 22 & - & - & 23.324 & 0.855 & - & - & - & - & - & - & - & - & - & - & - & - \\
\hline & - & - & & 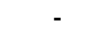 & - & - & - & - & 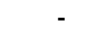 & - & - & - & - & - & - & - \\
\hline
\end{tabular}




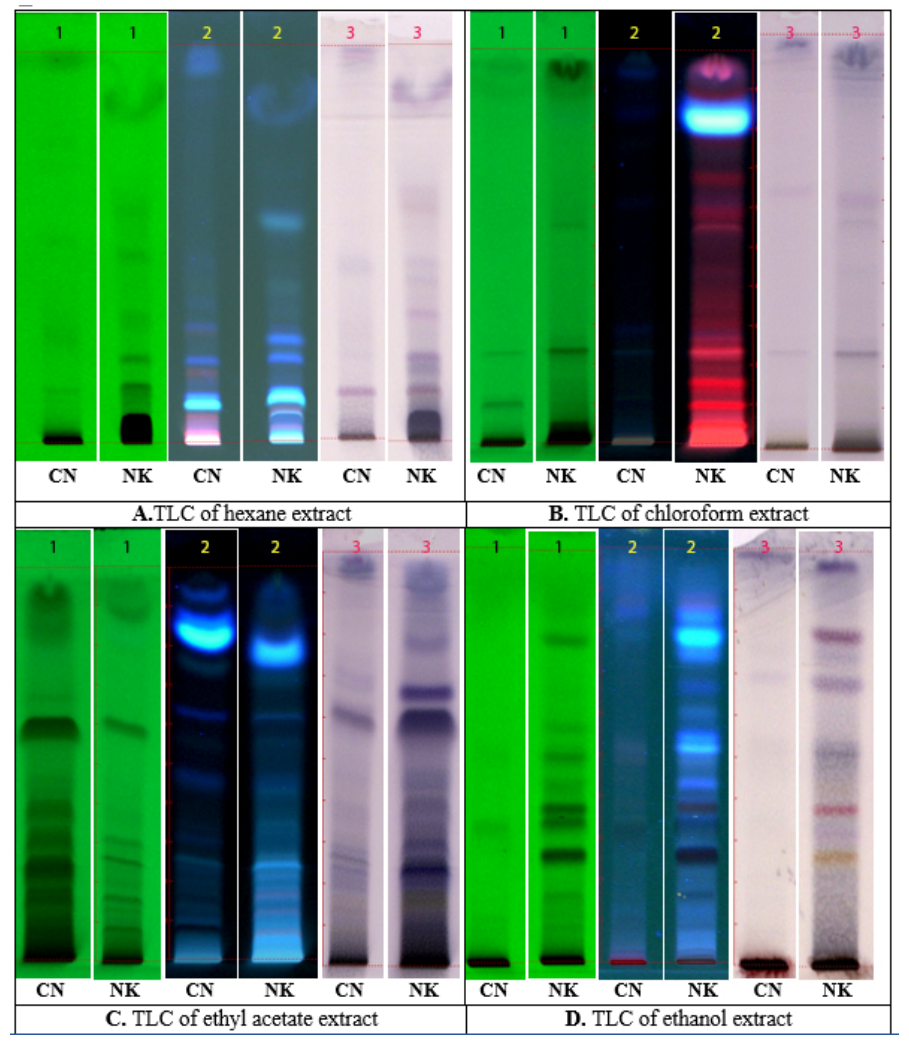

Fig. 1: TLC pattern of different extracts at $254 \mathrm{~nm}, 366 \mathrm{~nm}$, white light after derivatization with VSR

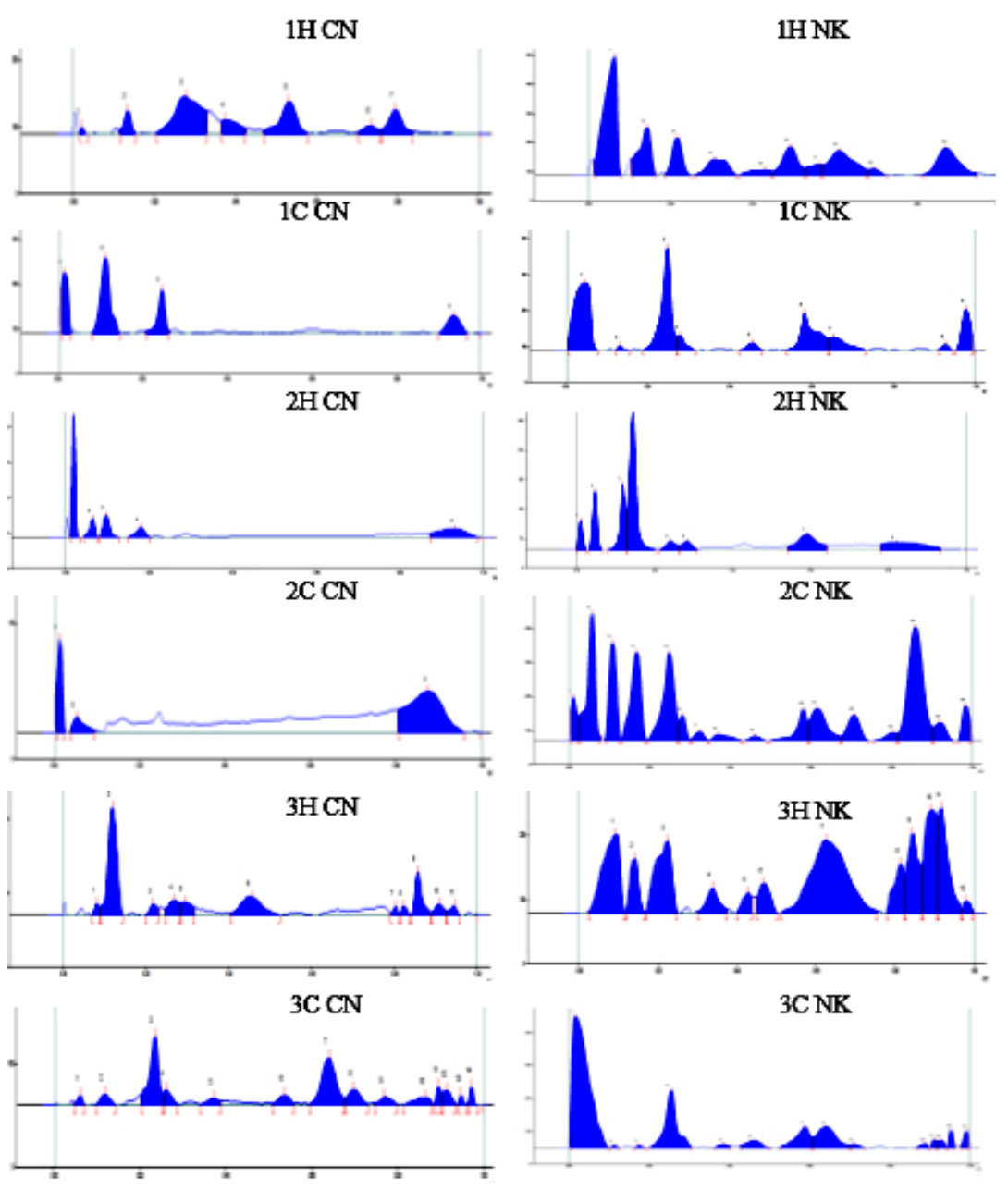

Fig. 2: Finger prints of hexane $(H)$ and chloroform $(C)$ extracts $(1-\lambda 254 ; 2-\lambda 366 ; 3-\lambda 520)$ 

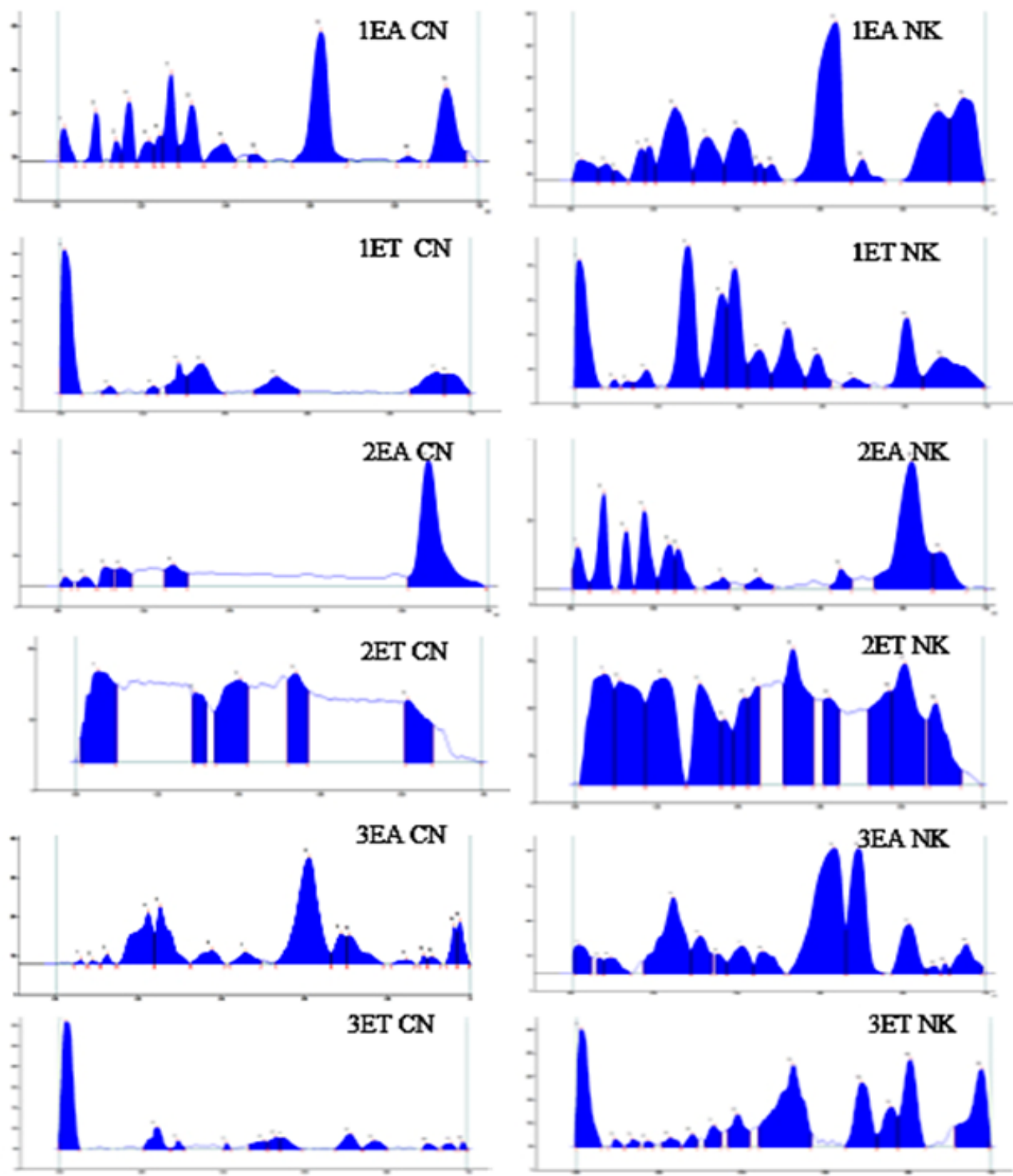

Fig. 3: Finger prints of Ethyl acetate (EA) and Ethanol (ET) extracts $(1-\lambda 254 ; 2-\lambda 366 ; 3-\lambda 520)$

separated peaks of hexane extract and ethanol extracts were monitored at $230 \mathrm{~nm}$; chloroform and ethyl acetate extracts were recorded at $200 \mathrm{~nm}$ (Table 1 and Table 2).

\section{RESULTS AND DISCUSSION}

The TLC photo documentation of all the successive extracts of Chennai Nagakesara/Cirunakappu $(\mathrm{CN})$ and Nagakesara (NK) are shown in fig. 1. TLC of hexane extract of $\mathrm{CN}$ showed spots at $\mathrm{R}_{\mathrm{f}} 0.13,0.27,0.52$ and 0.76 (all green) whereas NK showed 7 spots at $\mathrm{R}_{\mathrm{f}} 0.14$, $0.21,0.29,0.32,0.47,0.58$ and 0.83 (all green) under UV $254 \mathrm{~nm}$; CN showed 0.03, 0.06 (both pink), 0.09 (fluorescent blue), 0.12 (blue), 0.17 (pale pink), 0.21, 0.27 and 0.29 (all blue) while NK showed 9 spots at $R_{f}$ $0.02,0.04,0.07,0.11,0.21,0.26,0.32,0.38$ and 0.55 (all blue) under UV $366 \mathrm{~nm}$; after derivatization with VSR, CN showed 4 spots at $\mathrm{R}_{\mathrm{f}} 0.04$ (grey), $0.12,0.21$ and 0.44 (all purple) and NK showed spots at $R_{\mathrm{f}} 0.13$, $0.18,0.21,0.32,0.40,0.45,0.58,0.63$ and 0.87 (all purple).

Chloroform extract of $\mathrm{CN}$ showed two spots at $\mathrm{R}_{\mathrm{f}} 0.11$ and 0.23 (both green) while that of NK showed spots at $\mathrm{R}_{\mathrm{f}} 0.23$ and 0.55 (both green) under $254 \mathrm{~nm}$; $\mathrm{CN}$ showed spots at $\mathrm{R}_{\mathrm{f}} 0.12,0.24,0.29,0.60,0.76$ and 0.84 (all pale blue) NK showed 14 spots at $\mathrm{R}_{\mathrm{f}} 0.05,0.11,0.17,0.24$, $0.27,0.32,0.37,0.45,0.56,0.60,0.680 .77$ (all pink), 0.83 (Fluorescent blue) and 0.89 (pink) under $366 \mathrm{~nm}$; $\mathrm{CN}$ showed two spots at $\mathrm{R}_{\mathrm{f}} 0.23$ and 0.62 (both grey) while that of NK showed spots at $\mathrm{R}_{\mathrm{f}} 0.23$ (purple), 0.54 and 0.59 (both grey) after dipping in VSR as shown in fig. 2.

TLC of ethyl acetate extract of CN showed eight spots at $\mathrm{R}_{\mathrm{f}} 0.09,0.13,0.16,0.25,0.31,0.37,0.59$ and 0.88 
(all green) under UV $254 \mathrm{~nm}$; 0.08, 0.17, 0.26, 0.31, $0.47,0.63,0.75$ (all blue), 0.83 (fluorescent blue), 0.86 and 0.93 (both blue) under UV $366 \mathrm{~nm} ; 0.17,0.20$ (both grey), $0.23,0.26,0.28,0.60,0.68$ and 0.70 (all purple); at the same time NK showed twelve spots at $\mathrm{R}_{\mathrm{f}} 0.07$, $0.10,0.16,0.18,0.23,0.32,0.38,0.43,0.47,0.59,0.67$ and 0.84 (all green) at UV $254 \mathrm{~nm}$; ten spots at $\mathrm{R}_{\mathrm{f}} 0.08$, 0.13 (both pale pink), $0.17,0.22,0.25,0.35,0.44,0.56$, 0.63 (all pale blue) and 0.78 (fluorescent blue) UV 366 $\mathrm{nm}$; nine spots at $\mathrm{R}_{\mathrm{f}} 0.10,0.18$ (both grey) $0.24,0.30$, $0.39,0.45,0.59,0.66,0.78$ (all purple) after dipping in VSR as shown in fig. 3.

TLC of ethanol extract of CN showed 4 spots at $R_{f} 0.07$, $0.11,0.32$ and 0.50 (all green) whereas NK showed
8 spots at $\mathrm{R}_{\mathrm{f}} 0.09,0.16,0.25,0.33,0.43,0.50,0.56$ and 0.78 (all green) under UV $254 \mathrm{~nm}$; CN showed 5 spots at $\mathrm{R}_{\mathrm{f}} 0.33$ (brown), 0.49, 0.53,0.85,0.87(all pink) while NK showed 12 spots at $R_{f} 0.09,0.17$ (ash), 0.26 (black), 0.38 (brown), 0.43 (violet), 0.52, 0.54 (sky blue), 0.61 (greenish blue), 0.67, 0.75 (blue), 0.79 (sky blue) and 0.85 (blue) under UV $366 \mathrm{~nm}$; after derivatization with VSR, CN showed 4 spots at $R_{f} 0.04$ (grey), 0.12, 0.21 and 0.44 (all purple) and $\mathrm{NK}$ showed spots at $\mathrm{R}_{\mathrm{f}} 0.13$, $0.18,0.21,0.32,0.40,0.45,0.58,0.63$ and 0.87 (all purple).

In the HPTLC finger print profile of hexane extract under UV $254 \mathrm{~nm}$ of $\mathrm{CN}$, the peaks at $\mathrm{R}_{\mathrm{f}} 0.28$ $(42.34 \%)$ and $0.53(22.19 \%)$ are the major peaks

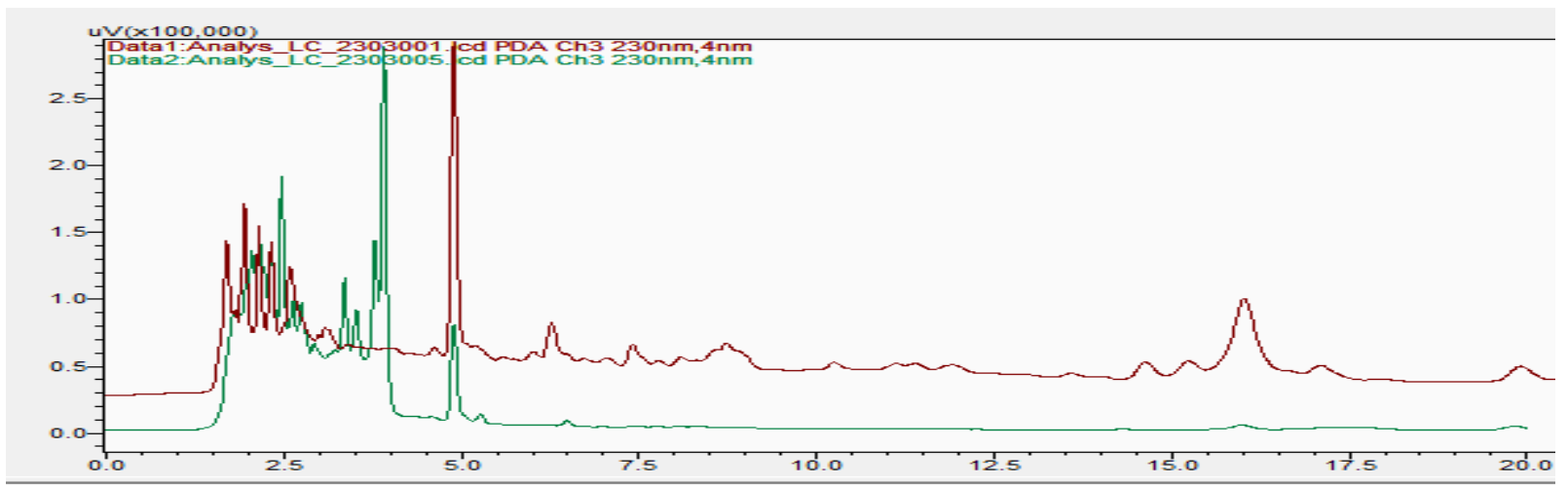

Fig. 4A: HPLC profile of hexane extract at $230 \mathrm{~nm}$

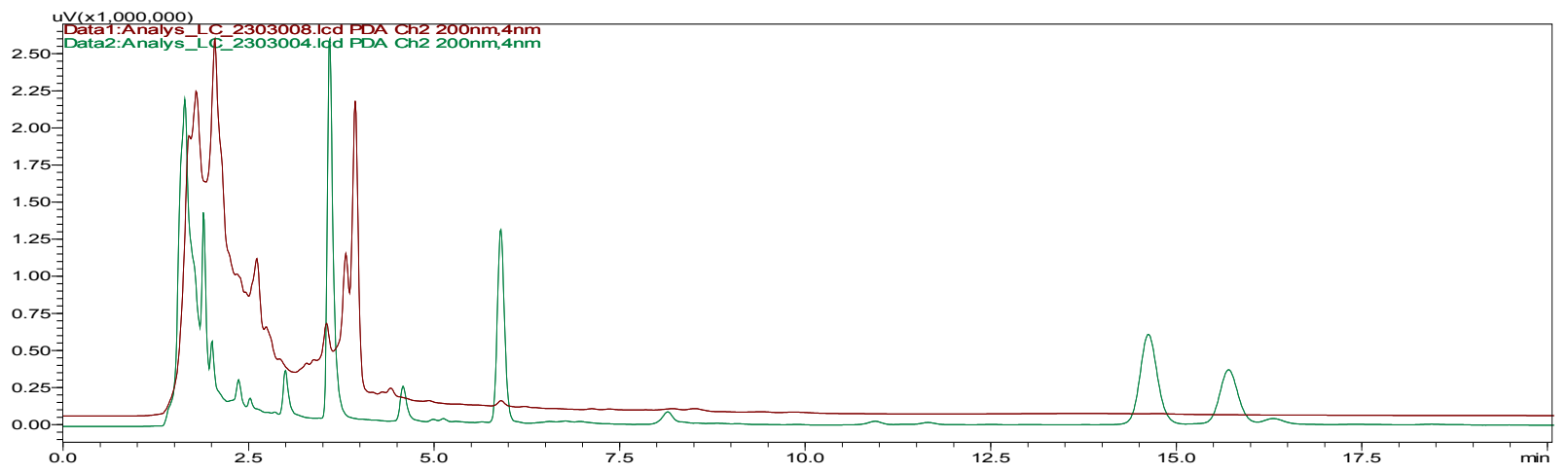

Fig. 4B: HPLC profile of chloroform extract at $200 \mathrm{~nm}$

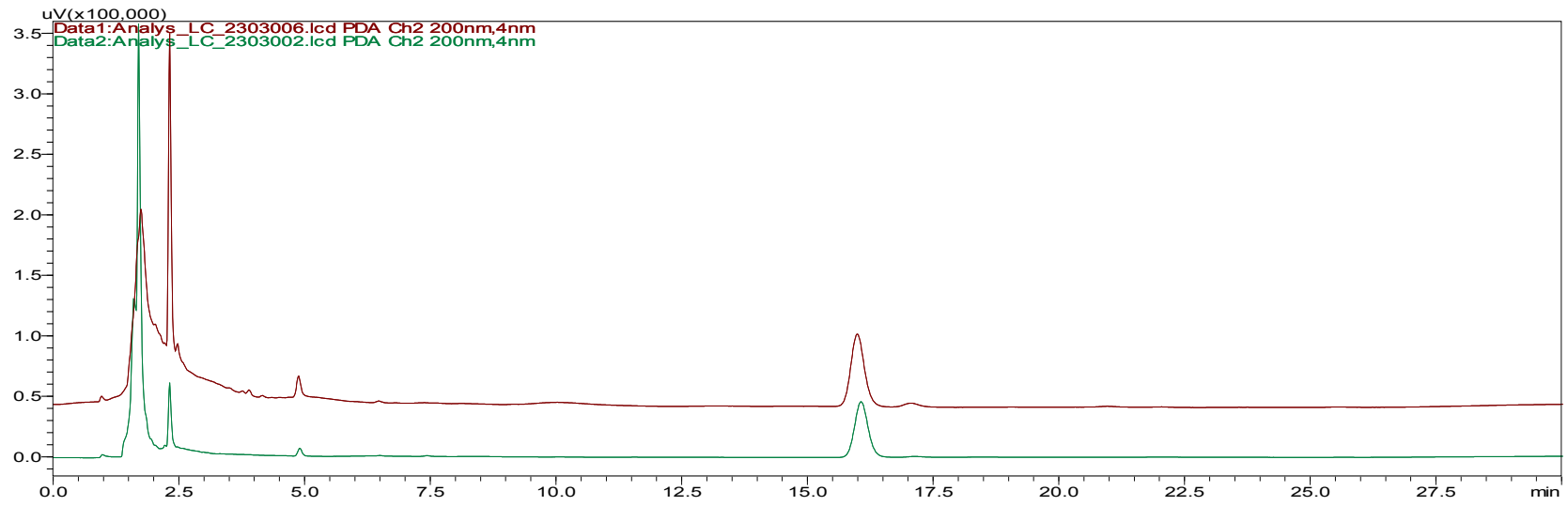

Fig. 4C: HPLC profile of ethyl acetate extract at $200 \mathbf{n m}$ 


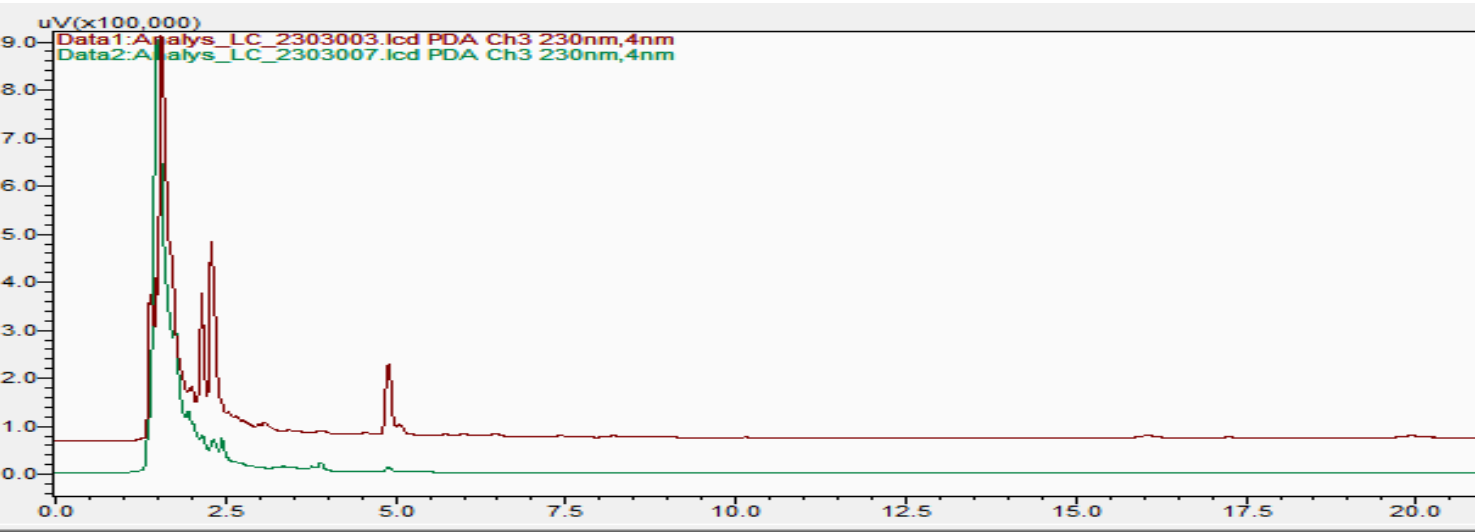

Fig. 4D: HPLC profile of ethanol extract at $230 \mathrm{~nm}$

but in $\mathrm{NK}$, the peaks at $\mathrm{R}_{\mathrm{f}} 0.61(13.04 \%)$ and $0.87(12.25 \%)$ are the major peaks; under $366 \mathrm{~nm}$ the peaks at $\mathrm{R}_{\mathrm{f}} 0.93(24.71 \%)$ and $0.02(41.43 \%)$ for $\mathrm{CN}$ and $0.14 \quad(39.28 \%), \quad 012 \quad(13.71 \%)$ for NK; after derivatization three major peaks were separated at $0.12 \quad(40.94 \%), \quad 0.46$ (16.96\%), $0.86(12.76 \%)$ for $\mathrm{CN}$ and at 0.22 (11.28\%), 0.62 (26.55\%) and 0.89 (12.03\%) for NK.

In the HPTLC finger print profile of chloroform extract under UV $254 \mathrm{~nm}$, three peaks separated at 0.08, 0.20 and 0.88 for $\mathrm{CN}$, eight peaks separated for NK in which peaks at $0.18(28.25 \%), 0.53(16.46 \%)$ and 0.93 $(10.06 \%)$ are major; under $366 \mathrm{~nm} 2$ peaks were separated in $\mathrm{CN}$ whereas in NK the peaks at 0.80 (23.17\%), $0.19(13.26 \%), 13(11.67 \%)$ were separated as major out of 15 peaks; after dipping in vanillinsulphuric acid 14 peaks and 13 peaks were separated in $\mathrm{CN}$ and NK respectively.

In the HPTLC finger print profile of ethyl acetate extract under UV $254 \mathrm{~nm}, 12$ peaks separated in which peaks at $0.55(31.38 \%), 0.86(18.96 \%), 0.25$ $(10.70 \%)$ are major in $\mathrm{CN}, 13$ peaks separated for NK in which peaks at $0.53(26.24 \%), 0.89$ (15.87\%), $0.78(14.37 \%)$ and $0.20(12.62 \%)$ are major; under $366 \mathrm{~nm} 5$ peaks were separated in $\mathrm{CN}$ whereas in NK the peaks at $0.72(42.20 \%), 0.05(10.53 \%), 0.15(10.27 \%)$ were separated as major out of 10 peaks; after dipping in vanillin-sulphuric acid 15 peaks in which 0.52 (39.51\%), $0.15(15.80 \%), 0.23(13.49 \%)$ and 14 peaks in which 0.51 (32.85\%), 0.65 (17.98\%), 0.17 (15.81 $\%$ ) were separated as major in $\mathrm{CN}$ and $\mathrm{NK}$ respectively.

In the HPTLC finger print profile of ethyl alcohol extract under UV $254 \mathrm{~nm}, 7$ peaks separated in which peaks at $0.31(16.39 \%), 0.85$ (12.54\%), 0.47 $(10.61 \%)$ are major in CN, 12 peaks separated for NK in which peaks at $0.22(19.89 \%), 0.37$ (14.37\%), 0.31 $(12.28 \%)$ and 0.85 (10.41\%) are major; under $366 \mathrm{~nm}$
4 peaks were separated in $\mathrm{CN}$ whereas in $\mathrm{NK}$ the peaks at $0.17(12.74 \%),(12.71 \%), 0.78(12.21 \%), 0.10$ $(11.74 \%)$ were separated as major out of 11 peaks; after dipping in vanillin-sulphuric acid 11 peaks in which $0.21(9.75 \%)$ and 12 peaks in which 0.44 $(25.80 \%), 0.78$ (14.09\%), 0.92 (15.01\%) were separated as major in $\mathrm{CN}$ and $\mathrm{NK}$ respectively.

The HPLC chromatograms of hexane, chloroform, ethyl acetate and ethanol extracts of $\mathrm{CN}$ and $\mathrm{NK}$ are shown in fig. 4A-fig. 4D. In the HPLC chromatogram of hexane extract at $230 \mathrm{~nm}, 14$ peaks in $\mathrm{CN}$ and 22 peaks in NK emerged in which the peaks at $\mathrm{R}_{t}$ 2.052 (13.04 \%), 3.908 (16.100\%) and 1.697 (10.285 \%), 2.589 (13.889 \%), 4.894 (15.951 \%, 16.020 $(14.627 \%)$ were the major peaks; in the chloroform extract at $200 \mathrm{~nm}, 13$ peaks in $\mathrm{CN}$ and 11 peaks in NK separated in which 1.611 (30.180\%), 3.526 (18.170\%), 5.788 (10.259\%), 14.355 (10.946\%) and $1.796(21.965 \%), 2.044$ (28.634 \%), 2.609 $(12.523 \%), 3.936(23.397 \%)$ respectively were the major peaks; in the ethyl acetate extract at $200 \mathrm{~nm}$, 5 peaks at 1.605 (21.842\%), 1.699 (42.469\%), 2.316 (16.035\%), 4.904 (0.929\%) 16.067 (18.724\%) in CN and 6 peaks at $1.744(45.980 \%), 2.316(16.513 \%), 2.471$ (18.733\%), 4.880 (4.955\%), 15.991 (13.060\%), 17.059 $(0.758 \%)$ in NK separated; in the ethyl alcohol extract, 17 peaks in which 1.489 (64.767\%), $1.758(14.337 \%)$ are major in $\mathrm{CN}$ and 5 peaks at 1.395 (9.672\%), 1.562 (59.321\%), 2.149 (9.135\%), 2.291 (16.766 \%), 4.894 $(5.107 \%)$ in NK separated. All these finger print profiles are unique to every extract of $\mathrm{CN}$ and $\mathrm{NK}$.

More than 300 medicinal plants have been listed as rare/red/threatened species and substitution for these herbs is the need of the hour ${ }^{[21]}$. The most common criteria for adulteration is the morphological similarity between the species; secondly economic aspects. The rare/red/threatened species would be costlier and hence 
adulterated with the cheaper one. Substitutions of herbs accomplish many goals though basic idea is to provide similar therapeutic effect as that of original drug. It provided a greater scope for the physician to utilize herbs that are easily available, cost effective and most appropriate for the clinical condition. It is not that all adulterations are intentional malpractice as stated in many literatures. With our experience it is noted that the herbal drugs are adulterated unintentionally also. Suppliers are not conversant and unaware about their spurious supply. Major reasons are confusion in name, non-availability and lack of knowledge about authentic plant. Even scientific community and traditional physicians are unaware of it. Dried floral buds of M. ferrea Linn, dried fruits of Dillenia pentagyna Roxb and dried fruiting inflorescence of $C$. wightii Meissn are used as Nagakesara in different regions of India ${ }^{[22]}$.

Cirunakappu, one of the study drug belongs to the genus Cinnamomum. Cirunakappu refers the flower as anatomical part of the plant but in market immature fruits are sold. If Cinnamomum species is to be used as Cirunakappu then the drug must originate from a flower not fruit. Otherwise, Cirunakappu might be the misnomer name of tender fruit of $C$. wightii like the fruit of Cuminum cyminum being called as cumin seed ${ }^{[23,24]}$.

Now a days, herbal drug industries follow high quality standards using modern techniques and instruments to maintain their quality. World Health Organization (WHO), in its publication on quality standards for medicinal plant materials, recommends rejecting any batch of raw material, which has more than $5 \%$ of any other plant part of the same plant (e.g. stem in leaf drugs), nevertheless if they are derived from the authentic plant. Based on these standards, adulteration whether, intentional or unintentional, should be rejected. Also, suppliers and traders should be educated about the authentic sources.

The plant $M$. ferrea is used from the ancient time for its medicinal values. Most of the Ayurvedic and Siddha formulations prescribed for various diseases have Nagakeshara/Cirunakappu as one of the ingredient. But due to huge demand and unavailability of any medicinal plant, there is a common trend to use substitute herb for the morphological similarities or therapeutic value. In the name of Chennai Nagakeshara/Cirunakappu, C. wightii is sold out in the market. But in place of stamen of $M$. ferrea (Nagakeshara) similar looking flower but of $C$. wightii (Cirunakappu) might have been used as an adulterant.
Both the flower of $M$. ferrea (Nagakeshara) and the flower bud or the tender fruit of $C$. wightii (Chennai Nagakeshara/Cirunakappu) are entirely different in genus, morphology and chromatographic finger print profiling of chemical constituents. Hence the therapeutic value may also be different from each other. Pharmacological screening of both plants is necessary to scientifically prove the efficacy and use of latter species in place of former species.

\section{Acknowledgements:}

Authors are thankful to The Director General, Central Council for Research in Siddha and The Assistant Director I/c, Siddha Central Research Institute for their support and encouragement.

\section{Funding:}

Funded by Central Council for Research in Siddha under Intramural Research Project Scheme vide sanction no.28/2016-17 dated 26.09.2016.

\section{Conflict of interests:}

The authors declared no conflicts of interest.

\section{REFERENCES}

1. Muthalia M. Siddha Materia Medica (Medicinal Plants Division). Department of Homeopathy, Directorate of Indian Medicines, Chennai; 1998.

2. Suresh C, Chandra TR, Rajeev K, Keeta K, Deep KG. Pharmacognostical evaluation of nagakesara used in different parts of India. Int J Ayur Pharm Res 2015;3:46-51.

3. Cinnamomum ovalifolium Gardner ex Meisn. IBIS-Flora. Prodr 1864;15(1):11.

4. Maridass M, Victor B. Ethnobotanical uses of Cinnamomum species, Tamil Nadu, India. Ethnobot leafl 2008;2008(1):18.

5. Sriramavaratharajan V, Murugan R. Screening of Chemical Composition, in vitro Antioxidant, $\alpha$-Amylase and $\alpha$-Glucosidase inhibitory activities of the leaf essential oils of Cinnamomum wightii from different populations. Nat Prod Comm 2018;13(11):1539-42.

6. Patra KC, Kumar KJ, Suresh P. Standardization of a polyherbal Siddha formulation, Amukkara Choornam. Indian $\mathrm{J}$ Tradit Knowl 2009;8(3):449-52.

7. Saraswathy A, Devi SN, Chandran RP. Analgesic and antiinflammatory activity of Amukkara curanam. Indian J Pharm Sci 2009;71(4):442.

8. Jeganathan NS, Kannan K, Manavalan R, Vasanthi HR. Standardization of a siddha formulation amukkaracuranam by HPTLC. Afr J Trad Comp Alt Med 2008;5(2):131-40.

9. Garg S, Mishra A, Gupta R. Fingerprint profile of selected ayurvedic churnas/preparations: An overview. Altern Integr Med 2013;2(6):1.

10. Pandey RK, Swarnlata S, Saraf S. Development of fingerprint for single component analysis of an ayurvedic formulation (Sitopaladi Churna) by high performance liquid chromatography. Der Pharm Lett 2010;2(2):464-70. 
11. The Siddha Formulary of India. Part I. 1st ed. (English version). New Delhi: Department of AYUSH, Ministry of Health and Family Welfare, Government of India; 1979. p. 177.

12. The Siddha Formulary of India. Part II. 1st ed. (Tamil version). New Delhi: Department of AYUSH, Ministry of Health and Family Welfare, Government of India; 2011. p. 298.

13. Asif M, Jafari SF, Iqbal Z, Revadigar V, Oon CE, Majid ASA, et al. Ethnobotanical and phytopharmacological attributes of Mesua ferrea: A mini review. J App Pharm Sci 2017;7(4):24251.

14. Stevens PF. Mesua ferrea became M. nagassarium but has to be called M. ferrea Again (Clusiaceae). Taxon 1986;35(2):352-4.

15. Dassanayake MD, Fosberg FR, Clayton WD. Flora of Ceylon. Amerind Publishing Co Pvt. Ltd, New Delhi. 1980;1:497-504.

16. The Ayurvedic Pharmacopoeia of India. Part II. Vol. I. New Delhi: Department of ISM and H, Ministry of Health and Family Welfare, Government of India; 2007. p. 7-92.

17. The Ayurvedic Pharmacopoeia of India. Part II. Vol. II. New Delhi: Department of ISM and H, Ministry of Health and Family Welfare, Government of India; 2008. p. 5-87.

18. The Ayurvedic Pharmacopoeia of India. Part II, Vol. III. New
Delhi: Department of ISM and H, Ministry of Health and Family Welfare, Government of India; 2010. p. 29-85.

19. Lallo D, Sahu AN, Hemalatha S, Dubey SD. Pharmacognostical and phytochemical evaluation of Cinnamomum wightii Meissn. flowers. Indian J Nat Prod Resour 2012;3(1):33-9.

20. Laloo D, Sahu AN. Antioxidant activities of three Indian commercially available Nagakesar: An in vitro study. J Chem Pharm Res 2011; 3(1):277-83.

21. Huang H. Plant diversity and conservation in China: planning a strategic bioresource for a sustainable future. Bot J Linn Soc 2011;166(3):282-300.

22. Anandakumar A, Balasubramanian M, Muralidharan R. Nagakesara-a comparative pharmacognosy. Anc Sci Life 1986;5(4):263.

23. Arunachalam C, Maheshwari B, Nartunai G, Ilavarasan R, Kumar KN, Sathiyarajeswaran P. A pharmacognosy approach to the botanical source of a cinnamon fruit traded as nāgakeśara and sirunagappu in raw drug markets. Pharmacogn J 2019;11(1):81-7.

24. Shakila R. Identification of phytoconstituents of Cirunakappu by gas chromatography-mass spectrometry. Int J Green Pharm 2020;14(3):274-9. 